\title{
The View of Life and Death and Decision Support at the End of Life
}

\section{Satomi Nakagi}

School of Nursing, Department of Nursing, Himeji University, Japan

\section{Abstract}

\section{Publication History:}

In the midst of the problems of end-of-life care, changes in the view of life and death, and changes in social conditions, we have examined how medical professionals should support decision-making at the end of life. As the view of life and death diversifies with changes in the social structure, how can we receive the thoughts and wishes of patients and provide end-of-life care that can respond to decisions according to each view of life and death? The challenge is how to incorporate the view of life and death into the Advance Care Planning (ACP) process.
Received: September 06, 2021

Accepted: September 11, 2021

Published: September 13, 2021

\section{Keywords:}

Pandemic teaching, Hope, Online teaching, Emotional intelligence, Empowerment, Nursing, Adult learners

\section{Introduction}

In recent years, I have come to hear the term "multi-death society" frequently. "Multi-death society" refers to a society in which the number of deaths increases and the population decreases due to the increase in the number of elderly people. The peak of the dying society is predicted to be around 2040 [1]. Issues in a multi-death society generally include the problem of end-of-life care and changes in the view of life and death. In the midst of such changes in social conditions, we have examined the ideal way of decision-making support for medical professionals at the end of life.

\section{Advance Care Planning}

Advance Care Planning (ACP) is a process of end-of-life decisionmaking that is close to and supports the patient. ACP is the process of planning overall care from early to end-of-life, such as the choice of treatments such as surgery, anticancer and radiation therapy, and when to discontinue treatment as the condition progresses. An important theme is the selection of a place of medical treatment at the end of life. In the West, it is widespread because patients are well aware of their right to autonomously decide what they really want. In Japan as well, ACP efforts are drawing attention.

Even when it comes to confirming the intention at the end of life, there is a possibility that the person's intention can change and that the person cannot convey his / her intention. Therefore, according to the definition of the Ministry of Health, Labor and Welfare [2], ACP is "a process in which the person himself / herself repeatedly discusses medical care / care at the final stage of life with his / her family and medical / care team in advance". At the root of ACP is respect for one's own values and views of life and death.

\section{Japanese View of Life and Death}

Regarding the view of life and death of the elderly Japanese, a previous study [3] showed that life satisfaction is related to reducing the fear of death and that poor health is related to the fear of death. It was also shown that those who have faith in religion can positively catch death. Kishimoto [4] points out that the role of religion is to alleviate the fear of death by preaching the afterlife in order to escape the inevitability of death. However, in the case of Japanese, the proportion of people who have faith is extremely low compared to Western countries, and the influence of religion on how to deal with death may not be so large in Japanese. Previous study [3] showed a view of life and death peculiar to Japanese elderly people related to local customs and family. Furthermore, it is clarified that the attitude toward life directly leads to the way of thinking about one's own death.

\section{End-of-Life Decision Support}

Even in the statement of position regarding "end-of-life medical care and care for the elderly" [5], it is difficult to predict life expectancy because the end-of-life of the elderly is affected by various factors and progresses to multiple variability. It has also been pointed out that it is difficult to support decision-making regarding end-of-life care for the elderly such as teachers and their families. Specific support measures such as the use of tools for grasping intentions and conferences with medical teams have been tried as support to reach a better end in line with the intentions of the elderly, but the number of cases is still small and trial and error is being carried out. It was considered to be in the stage of systematization.

The author investigated the nurses' perception of ACP, the content of ACP implementation, the timing of confirmation of intention, the usefulness of the conference in the implementation of ACP, and the usefulness of the summary of hearing wishes for hospitalization. It has been shown that it is an issue to proceed with discussions within the medical team regarding the creation of tools such as interview guides to grasp the wishes of the elderly and the timing of working to confirm the intentions of the elderly rice field [6]. At that time, considering each person's view of life and death is also considered to be an important key to grasping the hope for the end of life.

\section{Conclusion}

In the clinical setting, we are still at the stage of groping for practical methods toward the concrete efforts of ACP. In the future, it is necessary to accumulate further results such as establishment of

"Corresponding Author: Prof. Satomi Nakagi, School of Nursing, Department of Nursing, Himeji University, Japan, E-mail: verisa323@gmail.com

Citation: Nakagi S (2021) The View of Life and Death and Decision Support at the End of Life. Int J Nurs Clin Pract 8: 345. doi: https://doi.org/10.15344/23944978/2021/345

Copyright: (C) 2021 Nakagi. This is an open-access article distributed under the terms of the Creative Commons Attribution License, which permits unrestricted use, distribution, and reproduction in any medium, provided the original author and source are credited. 
concrete methods, technical education for support, and environmental improvement. An increasing number of people want to reach the end of their lives at their familiar homes and long-term care facilities instead of hospitals. However, at present, there are problems such as the limitation of providing sufficient medical care to people at the end of life other than medical institutions and the shortage of visiting doctors and nurses.

As the view of life and death diversifies with changes in the social structure, how can we receive the thoughts and wishes of patients and provide end-of-life care that can respond to decisions according to each view of life and death? The challenge is how to incorporate the view of life and death into the ACP process.

\section{Competing Interests}

The author declare that there is no competing interests regarding the publication of this article.

\section{Acknowledgments}

This paper is a part of the results implemented with the support of the Grant-in-Aid for Scientific Research (19K19751) of the Japan Society for the Promotion of Science.

\section{References}

1. National Institute of Population and Security (2020) Japan's Future Estimated Population.

2. Ministry of Health, Labor and Welfare (2018) Guidelines for the decision process of medical care and care at the final stage of life.

3. Nakagi S (2011) Factors Affecting Elderly People's Attitudes toward Death Japanese Clinical Thanatology 16: 67-78

4. Kishimoto H (2012) A heart that stares at death for ten years with cancer Kodansha, 1973.

5. The Japan Geriatrics Society: Statement of the position of the Japan Geriatrics Society regarding "medical care and care for the elderly at the end of life".

6. Nakagi S (2020) End-of-life decision-making support in home-visit nursing, Advance Care Planning (ACP) initiatives, current status and issues of practice for home-based elderly people, Nursing and Health Science Research Journal.
This article was originally published in a special issue:

Adult and Gerontological Nursing: Practices and Care

Handled by Editor(s):

Prof. Hiroko Shimizu

Department of Nursing

Kagawa University

Japan 